\title{
The Travelling Wave Solutions of the nonlinear beam equations
}

\author{
Weishi Yin ${ }^{1}$, Yixian $\mathrm{Gao}^{2}$, Pinchao meng ${ }^{1 *}$
}

${ }^{1}$ Shool of Science, Changchun university of Science and Technology, Jilin 130022,P.R.China

${ }^{2}$ Shool of Mathematic and Statistic, Northeast Normal university of Science, Jilin 130024,P.R.China Xuexi123@163.com

Keywords: Traveling wave solutions; Tanh-function method; Nonlinear Beam equation.

Abstract. This paper is concerned with the existence of exact traveling wave solutions of nonlinear evolution equation by the tanh-function method. The validity and reliability of this method is demonstrated by applying it to a variety of the nonlinear beam equations.

\section{Introduction}

It is well known that nonlinear phenomena are very important in a variety of scientific fields, especially in fluid mechanics, solid state physics, plasma physics, plasma waves, capillary-gravity waves, and chemical physics. Most of these phenomena are described by nonlinear partial differential equations. Analytical solutions of this problems are usually not available, especially when the nonlinear terms are involved. Therefore, finding its traveling solutions is of practical importance.

The methods of looking for exact traveling wave solutions of nonlinear evolution equations, has been tremendous development in recent decades, such as inverse scattering method ${ }^{[1]}$, Hirota's bilinear technique $^{[5]}$, the Painlve expansion method ${ }^{[12]}$.In the early nineties of last century, Huibin and Kelin ${ }^{[6]}$ proposed a new method. The main idea of this method is taking hyperbolic tangent function of the power series as possible traveling wave solutions of the nonlinear evolution equations. In order to reduce the complex algebra computation, Malfiety ${ }^{[8-10]}$ proposed the tanh-function method. Fan et al $^{[3]}$ proposed the extended hyperbolic tangent method, which replace the tanh-function by the solutions of Riccati equation. $\operatorname{In}^{[1,4,13]}$, using the tanh function method, they got the exact form of traveling wave solutions of various types of evolution equations.

\section{The Tanh-function Method}

Let's consider the nonlinear partial differential equations

$$
N\left(u, u_{x}, u_{x x}, u_{x x x}, \ldots\right)=0 \text {, }
$$

Where $u(x, t)$ is the real function on $R^{2}$. At first, we assume the traveling wave solutions of (2.1) are the form of

$$
u(x, t)=U(\omega)=U(c(x-v t)),
$$

with the velocity $v$, and the constant $c$. Submitted (2.2) into (2.1), we can get the ODES abou $\omega$

$$
N\left(U, U^{\prime}, U^{\prime \prime}, U^{\prime \prime \prime}, \ldots\right)=0,
$$

Second, we assume the possibly traveling wave solutions can be written

$$
u(x, t)=U(\omega)=H(Y)=\sum_{i=0}^{K} a_{i} Y^{i},
$$

where $Y=\tanh (\omega)=\frac{e^{\omega}-e^{-\omega}}{e^{\omega}+e^{-\omega}}$, the highest order $K$ will be determined late. Then we can get

$$
\frac{d Y}{d \omega}=1-Y^{2}, \frac{d U}{d \omega}=(1-Y) H^{\prime}, \frac{d^{2} U}{d \omega^{2}}=\left(1-Y^{2}\right)\left(-2 Y \cdot H^{\prime}+\left(1-Y^{2}\right) H^{\prime \prime}\right)
$$




$$
\left.\frac{d^{3} U}{d \omega^{3}}=\left(1-Y^{2}\right)\left(6 Y^{2}-2\right) H^{\prime}-6 Y\left(1-Y^{2}\right) \cdot H^{\prime \prime}+\left(1-Y^{2}\right)^{2} H^{\prime \prime \prime}\right),
$$

Submitted above equations into (2.3), we can get the ODES with $\mathrm{Y}$

$$
N\left(U, U^{\prime}, U^{\prime \prime}, U^{\prime \prime \prime}, \ldots\right)=0 \text {, }
$$

where $H^{\prime}=\frac{d H}{d Y}$. To determine the parameter $K$ we usually balance the nonlinear term and the highest order derivative term in equation (2.5).Then, we submitted (2.4) (with the determined $K$ ) into (2.5), and get the polynomial equation with $Y$. Collecting all the coefficients of power of $Y$ and letting the coefficients of each power of $Y$ to be vanished, we can determined all the coefficient $a_{1}, a_{2}, \ldots, a_{K}$. According to (2.4), we can get the traveling wave solutions of (2.1).

\section{Application}

Let us consider the traveling wave solutions of the nonlinear beam equations

$$
u_{t t}=u_{x x x x}+u\left(1-u^{2}\right) \text {. }
$$

Submitting (2.2)and (2.4)into(3.1), we can get

$$
\begin{aligned}
& \left.c^{2} v^{2}\left(1-Y^{2}\right)\left(-2 Y H^{\prime}\right)+\left(1-Y^{2}\right) H^{\prime \prime}\right)=c^{4}\left(1-Y^{2}\right)\left(8 Y\left(2-3 Y^{2}\right)\right) H^{\prime} \\
& \left.-2\left(4-13 Y+3 Y^{4}\right) H^{\prime \prime}+3\left(1-Y^{2}\right)\left(1-Y^{2}-2 Y\right) H^{\prime \prime \prime}+\left(1-Y^{2}\right)^{3} H^{(4)}\right)+H\left(1-H^{2}\right) .
\end{aligned}
$$

Balancing $Y^{8} H^{(4)}$ with $H^{4}$, we can get $K=2$. Thus

$$
H(Y)=a_{0}+a_{1} Y+a_{2} Y^{2} \text {. }
$$

Submitting it into above equation, we can get

$$
\begin{aligned}
& c^{2} v^{2}\left(1-Y^{2}\right)\left(-2 Y\left(a_{1}+2 a_{2} Y\right)+2\left(1-Y^{2}\right) a_{2}\right)=c^{4}\left(1-Y^{2}\right) \\
& \left(8 Y\left(2-3 Y^{2}\right)\left(a_{1}+2 a_{2} Y\right)-4 a_{2}\left(4-13 Y+3 Y^{4}\right)\right)+\left(a_{0}+a_{1} Y+a_{2} Y^{2}\right)\left(1-\left(a_{0}+a_{1} Y+a_{2} Y^{2}\right)^{2}\right) .
\end{aligned}
$$

$\mathrm{g}$ the coefficient of the same power of $Y$, we can get

$$
\left\{\begin{array}{c}
a_{0}-a_{0}^{3}-16 c^{4} a_{2}-2 c^{2} v^{2} a_{2}=0, \\
a_{1}+16 c^{4} a_{1}+2 c^{2} v^{2} a_{1}-3 a_{0}^{2} a_{1}=0, \\
-3 a_{0} a_{1}^{2}+a_{2}+100 c^{4} a_{2}+8 c^{2} v^{2} a_{2}-3 a_{0}^{2} a_{2}=0, \\
-40 c^{2} a_{2}-2 c^{2} v^{2} a_{1}-a_{1}^{3}-6 a_{0} a_{1} a_{2}=0, \\
-144 c^{4} a_{2}-6 c^{2} v^{2} a_{2}-3 a_{1}^{2} a_{2}-3 a_{0} a_{2}^{2}=0, \\
24 c^{4} a_{1}-3 a_{1} a_{2}^{2}=0, \\
60 c^{4} a_{2}-a_{2}^{3}=0 .
\end{array}\right.
$$

Then, we can get

$$
\begin{aligned}
& a_{1}=0, a_{0}=-\frac{\sqrt{\frac{15}{2}}}{2}, v=-\frac{\sqrt{3}}{2^{\frac{3}{4}}}, a_{2}=\frac{\sqrt{\frac{15}{2}}}{2}, c=-\frac{1}{2^{\frac{4}{5}}} ; \\
& a_{1}=0, a_{0}=-\frac{\sqrt{\frac{15}{2}}}{2}, v=\frac{\sqrt{3}}{2^{\frac{3}{4}}}, a_{2}=\frac{\sqrt{\frac{15}{2}}}{2}, c=-\frac{1}{2^{\frac{4}{5}}} ; \\
& a_{1}=0, a_{0}=\frac{\sqrt{\frac{15}{2}}}{2}, v=-\frac{\sqrt{3}}{2^{\frac{3}{4}}}, a_{2}=-\frac{\sqrt{\frac{15}{2}}}{2}, c=-\frac{1}{2^{\frac{4}{5}}} ; \\
& a_{1}=0, a_{0}=\frac{\sqrt{\frac{15}{2}}}{2}, v=\frac{\sqrt{3}}{2^{\frac{3}{4}}}, a_{2}=-\frac{\sqrt{\frac{15}{2}}}{2}, c=-\frac{1}{2^{\frac{4}{5}}} .
\end{aligned}
$$




$$
\begin{aligned}
& \text { Then, We can get } \\
& \left\{\begin{array}{l}
u_{1}(x, t)=-\frac{\sqrt{\frac{15}{2}}}{2}+\frac{\sqrt{\frac{15}{2}}}{2} \tanh ^{2}\left[\frac{1}{2^{\frac{5}{4}}}\left(x+\frac{\sqrt{3}}{2^{\frac{3}{4}}} t\right)\right] \\
u_{2}(x, t)=-\frac{\sqrt{\frac{15}{2}}}{2}+\frac{\sqrt{\frac{15}{2}}}{2} \tanh ^{2}\left[\frac{1}{2^{\frac{5}{4}}}\left(x-\frac{\sqrt{3}}{2^{\frac{3}{4}}} t\right)\right] \\
u_{3}(x, t)=\frac{\sqrt{\frac{15}{2}}}{2}-\frac{\sqrt{\frac{15}{2}}}{2} \tanh ^{2}\left[\frac{1}{2^{\frac{5}{4}}}\left(x+\frac{\sqrt{3}}{2^{\frac{3}{4}}} t\right)\right] \\
u_{4}(x, t)=\frac{\sqrt{\frac{15}{2}}}{2}-\frac{\sqrt{\frac{15}{2}}}{2} \tanh ^{2}\left[\frac{1}{2^{\frac{5}{4}}}\left(x-\frac{\sqrt{3}}{2^{\frac{3}{4}}} t\right)\right]
\end{array}\right.
\end{aligned}
$$
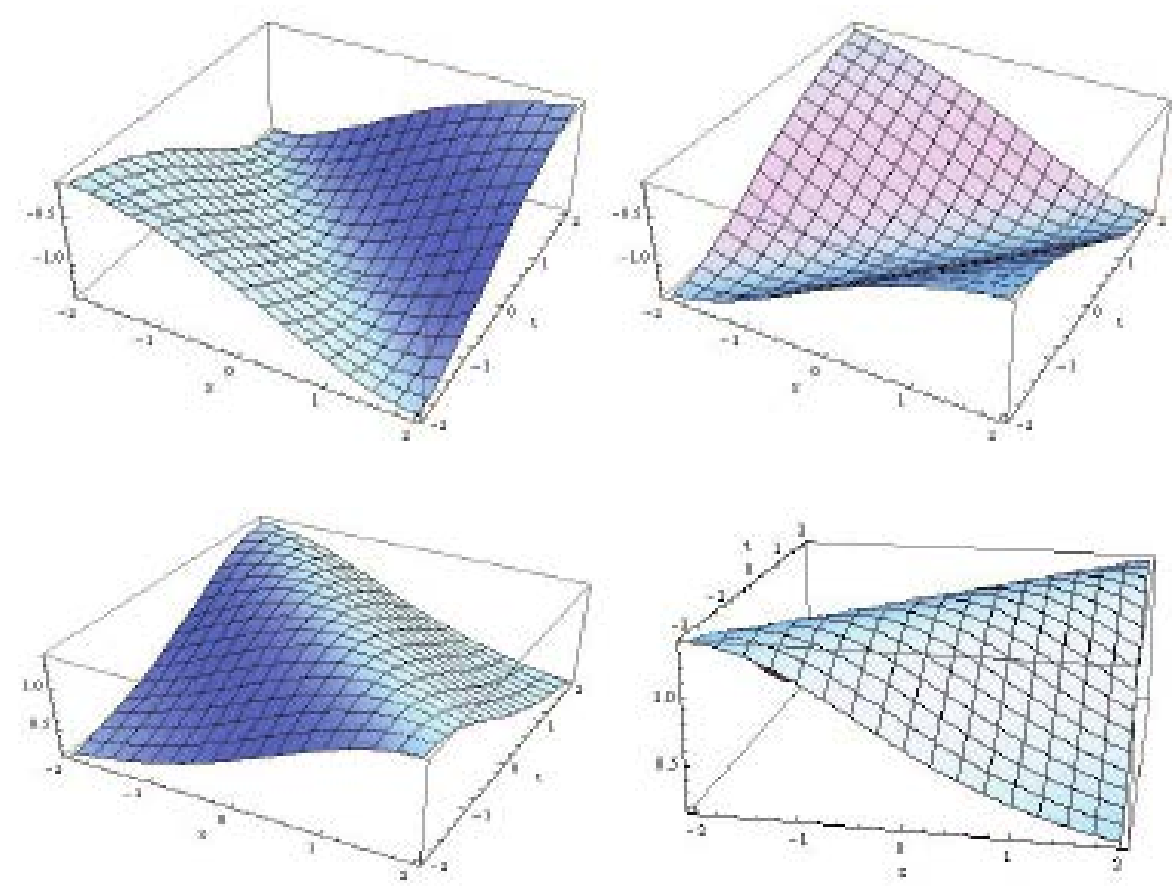

Fig. 1. the travelling wave solutions of $(3.2)$, when $(x, t) \in[-2,2] \times[-2,2]$.

\section{References}

[1] M.A. Abdou, New exact travelling wave solutions using modified extended tanh-function method, Chaos, Solitons and Fractals 31 (2007), 840-85.

[2] M.J. Ablowitz, P.A. Clarkson, Solitons, Nonlinear Evolution and Inverse Scattering, Cambridge Univ. Press, 1991.

[3] E. Fan, Y. Hon, Generalized tanh method extended to special types of nonlinear equations,Z. Naturforsch, 57 (2002) 692-700.

[4] K. Glasner, Nonlinear preconditioning for diffuse interfaces, J. Comput. Phys. 174 (2001),695-711.

[5] C.H. Gu, Soliton Theory and Its Application, Springer, Berlin, 1995.

[6] L. Huibin, W. Kelin, Exact solutions for two nonlinear equations: I, J. Phys. A: Math. Gen.23(1990), 3923-3928

[7] A.H. Khater, W. Malfliet, D. K. Callebaut, E. S. Kamel, The tanh method, a simpletransformation and exact analytical solutions for nonlinear reaction-diffusion equations, Chaos Soliton. Fract 14 (2002), 513-522. 
[8] W. Malfliet, Solitary wave solutions of nonlinear wave equations, Am. J. Phys. 60 (7)(1992), 650-654.

[9] W. Malfliet, The tanh method: I. Exact solutions of nonlinear evolution and wave equations,Physica Scripta 54 (1996), 563-568.

[10] W. Malfliet, The tanh method: II. Perturbation technique for conservative systems, Physical Scripta, 54 (1996), 569-575.

[11] E.J. Parkes, B.R. Duffy, An automated tanh-function method for finding solitary wave solutions to non-linear evolution equations, Comput. Phys. Commun 98 (1996), 288-300.

[12] B.Tian, Y. Gao, Truncated Painleve expansion and a wide-ranging type of generalized variable-coefficient Kadomtsev-Petviashvili equations, Phys. Let. A 209 (1995), 297-304.

[13] M.Wang, Exact solutions for a compound KdV-Burgers equation, Phys. Lett. A 213 (1998),279-287.5. 